\title{
On Magnetic Plucking Configurations for Frequency Up-Converting Mechanical Energy Harvesters
}

\author{
Tiancheng Xue* and Shad Roundy \\ Department of Mechanical Engineering, University of Utah, Salt Lake City, UT 84112, USA
}

\begin{abstract}
Magnetic plucking applies the strategy of frequency up-conversion in inertial energy harvesting when the energy source, such as human motion, provides excitations with very low and irregular frequencies. In a typical implementation, a slower moving inertial mass magnetically plucks a piezoelectric cantilever beam which converts mechanical energy to electrical energy at a higher frequency. We categorize several feasible magnet configurations to achieve plucking. We classify these as either inplane (the beam is deflected in the plane of proof mass motion) or out-of-plane (the beam is deflected orthogonal to the plane of proof mass motion). Whereas in-plane plucking induces a clean ring down due to its inherent jump phenomenon, out-ofplane plucking enables the capability of fabricating multiple piezoelectric beams on a single substrate. This paper presents an analysis of three different out-of-plane plucking configurations along with the in-plane repulsive configuration based on a three-dimensional analytical cube permanent magnet model. We derive a magnetically plucked piezoelectric beam model to investigate the dynamic characteristic for different plucking configurations. After validating the model with experimental results we extend the simulation into a larger driving frequency domain to compare two types of magnet configurations in terms of power generation.
\end{abstract}

\section{Introduction}

Mechanical energy harvesters convert ambient vibration into electricity to power sensors and electronics as an alternative to conventional batteries when energy independence is preferred. In general, these harvesters apply one of three basic transduction mechanisms to achieve the electromechanical conversion: electrostatic, electromagnetic, or piezoelectric. The feasibility of using energy harvesters to power wireless sensor nodes has been validated[1-3] and industrial applications such as self-powered tire pressure sensors have been realized[4].

In recent years, there has been a growing interest in studying energy harvesting from human motion in the research community due to the emerging market for wearables. Currently, commercially available wearable wellness products all suffer from the hassle of needing to frequently recharge the batteries. Wearable energy harvesting could provide the potential for continuous $24 / 7$ health monitoring which is crucial for some clinical applications such as electrocardiographic (ECG) monitoring[5]. We categorize human motion into inertia motion, in which the human motion excites an inertial mass from which power is generated, and joint motion, in which energy is harvested directly from the rotation of a joint such as the knee. In the case of joint motion, a conventional

\footnotetext{
* Corresponding author, Email: tiancheng.xue@utah.edu
} 
electric generator based harvester has been demonstrated in [6] to generate power from the knee joint during human gait. The prototype is considerably bulky $(790 \mathrm{~g})$ as a wearable device. As the size of the harvester reduces, piezoelectric transducers becomes more practical. This is especially true if gearing systems can't be used which are usually necessary to increase the voltage from electromagnetic harvesters. Wearable electronic devices are typically designed as wrist bands or chest bands. At these body locations the inertia motion is more relevant. However, the inherent limitation of utilizing human motion as the source for inertial energy harvesting is that it only provides excitations with very low and irregular frequencies. Typically, a linear oscillator based energy harvester operates at its natural frequency to take advantage of the peak dynamic magnification. At very low frequencies, this requires very large proof mass motions, which are not feasible for wearable energy harvesters. Furthermore, the very slow and large amplitude proof mass motion does not directly actuate a transducer very effectively. For example, as mentioned earlier, the slow speed results in very low voltages from an electromagnetic transducer. Furthermore, a large amplitude motion would typically require unfeasibly large piezoelectric displacements. Thus a standard linear energy harvester suffers from low effectiveness[7] in the case of wearables. Frequency up-conversion is a commonly applied strategy to tackle this issue by transforming the low-frequency input motion into a highfrequency actuation of the transducer. In terms of piezoelectric energy harvesters, plucking a cantilever beam is one technique that applies such a strategy. Eccentric rotor-based wearable energy harvesters have been demonstrated in the literature either using magnets[8-10] or pins[11,12] to pluck piezoelectric beams. Depending on the location, the target power output of these harvesters are either a few dozen microwatts (wrist) or milliwatts (knee joint). In these harvesters, the proof mass is a semicircular rotor, such as those originally used in self-winding automatic and motion-powered quartz watches[13,14]. As shown in FIG. 1, the rotational proof mass can be excited in all directions with no inherent motion limit, which caters to the irregular nature of human motion. Compared to mechanical plucking, the use of magnetic coupling to pluck the piezoelectric beams provides better reliability since it can be designed to be contact-free. Usually magnets are arranged to pluck the beam in the direction of magnet motion as shown in FIG. 2 (a), which is defined as in-plane plucking. Whereas the in-plane plucking introduces a jump phenomenon to cause ring down in the cantilever beam, another configuration, the out-of-plane plucking provides some alternative advantages, making it worth investigation. In addition, a better understanding of the 
plucked beam dynamics will help improve the performance of the plucked piezoelectric beam-based energy harvesters.

We present three alternative out-of-plane magnetic plucking configurations in this paper: the direct repulsive configuration (DRC), the orthogonal configuration (OC), and the indirect repulsive configuration (IRC). As shown in FIG. 2. (b)(c)(d), the beam deflection direction is perpendicular to the plane of magnet motion in the out-of-plane plucking configurations. This arrangement enables the possibility of fabricating multiple beams on a single substrate[15] which reduces assembly complexity when multiple beams are embedded in the device to achieve higher power output. In addition, it has better MEMS process compatibility when the device is scaled down.

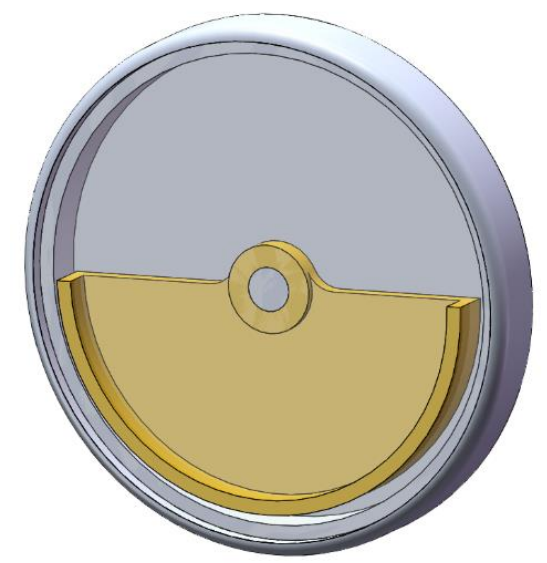

FIG. 1. Eccentric rotor-based energy harvester

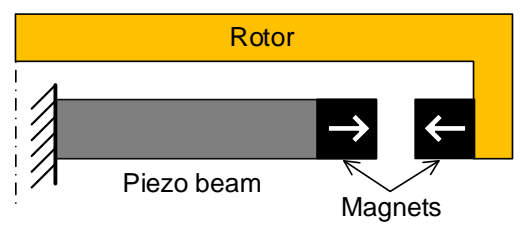

(a)

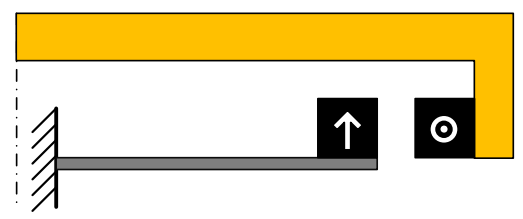

(c)

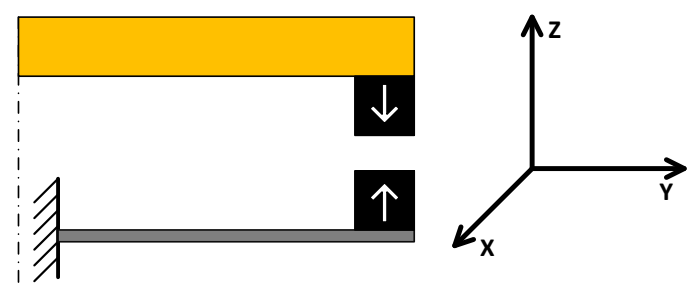

(b)

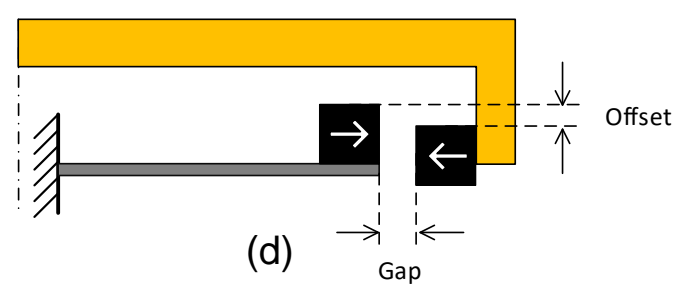

FIG. 2. Magnetic plucking configurations in section view: (a) in-plane plucking configuration, (b) out-of-plane direct repulsive configuration (DRC), (c) out-of-plane orthogonal configuration (OC), (d) out-of-plane indirect repulsive configuration (IRC). The moving magnet is attached on the rotor and moves in the $x$ direction at this instance. In (a), the beam bends in the $x$ direction whereas in (b) (c) (d), it bends in the $z$ direction. 
This work expands upon a previous analysis on magnetic plucking configurations based on static magnetic force profiles[15] (i.e. interaction between one fixed magnet and one moving magnet). We have augmented the model into a full dynamic magnetic plucking system based on a distributed parameter piezoelectric bimorph beam model while incorporating magnetic actuation forces in both transverse and axial directions. A three-dimensional surface fitting technique can be employed to save computation time by simplifying the analytical magnet model in a dynamic simulation. Both simulation and experimental results will be presented and discussed with respect to implementation in frequency up-converting energy harvesting systems.

\section{Magnetic plucking configurations}

\subsection{Forces exerted between permanent magnets}

The fundamental idea behind magnetic plucking is to use magnetic coupling to create a force profile in the form of a narrow Gaussian function, mimicking an abrupt and clean release of the beam tip. Therefore, the quantitative determination of the magnetic interaction force is of great importance. In a degenerate case, permanent magnets can be simplified to magnetic dipoles when the sizes are negligible compared to the distance between them. In a more general case, however, it is difficult to obtain an accurate expression analytically for the interaction between permanent magnets. Nevertheless, some analytical solutions for simple geometries exist in the literature based on either the Coulombian charge model[16] or the Ampère's current model[17] with the assumption of constant and uniform polarization. In more recent work, Kelvin's formula has also been applied to derive closed-form solutions for forces acting on the magnet due to the field originated by a second magnet[18,19]. Although computationally expensive, finite element methods based on Maxwell's stress tensor or virtual work principle predict more reliable results[20] especially for complex geometries.

For many energy harvesting devices, the size of the magnets used for plucking piezoelectric beams is on the same order of magnitude as the distance. Thus, a simple magnetic dipole model is not appropriate. In addition, the magnetic torque is often overlooked in previous studies on magnetic plucking whereas it could potentially provide a nontrivial excitation[10,21]. We applied an analytical solution[16] in this study to obtain the static magnetic force profile as a starting point. Note that the aspect ratio, i.e., the ratio of the thickness to the width of a magnet for a 
given volume plays an important role in determining its strength. It has been shown that an aspect ratio of 1 falls in the optimal range to achieve maximum contact force per volume[19]. Therefore, cube magnets are chosen for this study. Granted the fact that modern rare-earth magnets exhibit good magnetization uniformity, a cube magnet can be modelled as two surfaces of evenly distributed magnetic charges[16]. The total potential energy between two cube magnets shown in FIG. 3 with parallel magnetization directions is given by:

$$
E=\frac{J J^{\prime}}{4 \pi \mu} \sum_{m=0}^{1} \sum_{n=0}^{1} \int_{-l}^{l} \int_{-l}^{l} \int_{-l}^{l} \int_{-l}^{l} \frac{(-1)^{m-n}}{\sqrt{\left(\alpha+X^{\prime}-X\right)^{2}+\left(\beta+Y^{\prime}-Y\right)^{2}+(\gamma+2(m-n) l)^{2}}} d X^{\prime} d Y^{\prime} d X d Y
$$

where $J$ and $J$ ' are the magnetic polarizations for each cube magnet of length $2 l$ and $\mu$ is the permeability of the medium. Note that here we assume uniform permeability whereas a difference does exist between air and magnetic material, which introduces a small error of less than $5 \% . O$ and $O$ ' are the center of the cube magnets. The origin of the $O^{\prime} X^{\prime} Y^{\prime} Z^{\prime}$ frame is located at $(\alpha, \beta, \gamma)$ with respect to the $O X Y Z$ frame (see FIG. 3). The forces acting on the fixed magnet originating at $O$ are the derivative of the potential energy with respect to each direction:

$$
F=-\frac{J J^{\prime}}{4 \pi \mu} \sum_{i=0}^{1} \sum_{j=0}^{1} \sum_{k=0}^{1} \sum_{l=0}^{1} \sum_{p=0}^{1} \sum_{q=0}^{1}(-1)^{i+j+k+l+p+q} \psi\left(\bar{U}_{i j}, \bar{V}_{k l}, \bar{W}_{p q}, \bar{R}\right)
$$

where

$$
\begin{gathered}
\psi_{x}(\bar{U}, \bar{V}, \bar{W}, \bar{R})=\frac{\left(\bar{V}^{2}-\bar{W}^{2}\right)}{2} \ln \left(\frac{\bar{R}-\bar{U}}{l_{0}}\right)+\bar{U} \bar{V} \ln \left(\frac{\bar{R}-\bar{V}}{l_{0}}\right)+\bar{V} \bar{W} \tan ^{-1}\left(\frac{\bar{U} \bar{V}}{\bar{W} \bar{R}}\right)+\frac{1}{2} \bar{U} \bar{R} \\
\psi_{y}(\bar{U}, \bar{V}, \bar{W}, \bar{R})=\frac{\left(\bar{U}^{2}-\bar{W}^{2}\right)}{2} \ln \left(\frac{\bar{R}-\bar{V}}{l_{0}}\right)+\bar{U} \bar{V} \ln \left(\frac{\bar{R}-\bar{U}}{l_{0}}\right)+\bar{U} \bar{W} \tan ^{-1}\left(\frac{\bar{U} \bar{V}}{\bar{W} \bar{R}}\right)+\frac{1}{2} \bar{V} \bar{R} \\
\psi_{z}(\bar{U}, \bar{V}, \bar{W}, \bar{R})=-\bar{U} \bar{W} \ln \left(\frac{\bar{R}-\bar{U}}{l_{0}}\right)-\bar{V} \bar{W} \ln \left(\frac{\bar{R}-\bar{V}}{l_{0}}\right)+\bar{U} \bar{V} \tan ^{-1}\left(\frac{\bar{U} \bar{V}}{\bar{W} \bar{R}}\right)-\bar{W} \bar{R}
\end{gathered}
$$

where $l_{0}$ is the unit rate constant to keep the argument of the logarithm function dimensionless, $\bar{U}, \bar{V}, \bar{W}$, and $\bar{R}$ are geometric parameters given by:

$$
\bar{U}_{i j}=\alpha+\left((-1)^{j}-(-1)^{i}\right) l
$$




$$
\begin{gathered}
\bar{V}_{k l}=\beta+\left((-1)^{l}-(-1)^{k}\right) l \\
\bar{W}_{p q}=\gamma+\left((-1)^{q}-(-1)^{p}\right) l \\
\bar{R}=\sqrt{\bar{U}_{i j}^{2}+\bar{V}_{k l}^{2}+\bar{W}_{p q}^{2}}
\end{gathered}
$$

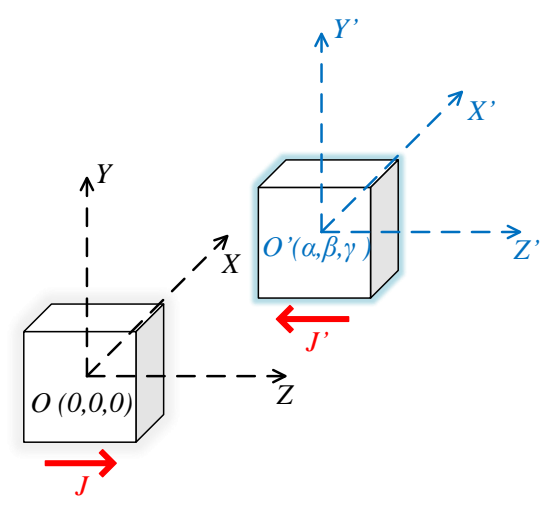

FIG. 3. Schematic of the generic magnetic configuration with repelling magnets.

A similar formula can be derived for the case where the magnetic polarizations are perpendicular or even arbitrarily oriented[22]. The magnetic torque components can be calculated as well with respect to each axis. We applied the analytical expressions above to obtain the interactions between magnets for the different magnetic plucking configurations shown earlier in FIG. 2. The contribution of the torque to the dynamics compared with the force depends on the length of the cantilever beam. For a cantilever beam longer than $10 \mathrm{~mm}$ as used in our study, the contribution of the torque is more than an order of magnitude smaller than the contribution of the force. Thus, we have neglected the torque components. As illustrated in , the static force profile is given as a function of the magnet displacement normalized by the length of magnet $2 l$. The force is normalized to be 1 at its maximum for the transverse component in the direct repulsive configuration (i.e., $f_{z}$ ). The moving magnet on the rotor is assumed to follow a linear path as the small angles between the opposing surfaces of the magnets can be neglected. In this example the length of the $\mathrm{N} 50$ magnets is $2 \mathrm{~mm}$ and the gap between them is $1 \mathrm{~mm}$. A $1 \mathrm{~mm}$ offset in the $z$ direction is applied in the indirect repulsive configuration. Note that an offset in the $z$ direction is essential to the indirect repulsive configuration as there will be no transverse force without it. We used the boundary integral method based 
Radia software[23] developed by the European Synchrotron Radiation Facility (ESRF) to provide a numerical validation for the analytical model.

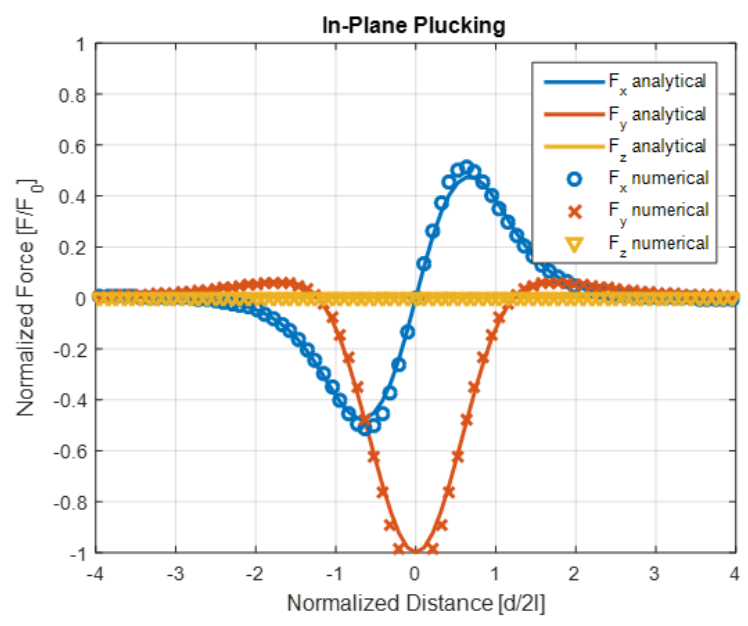

(a)

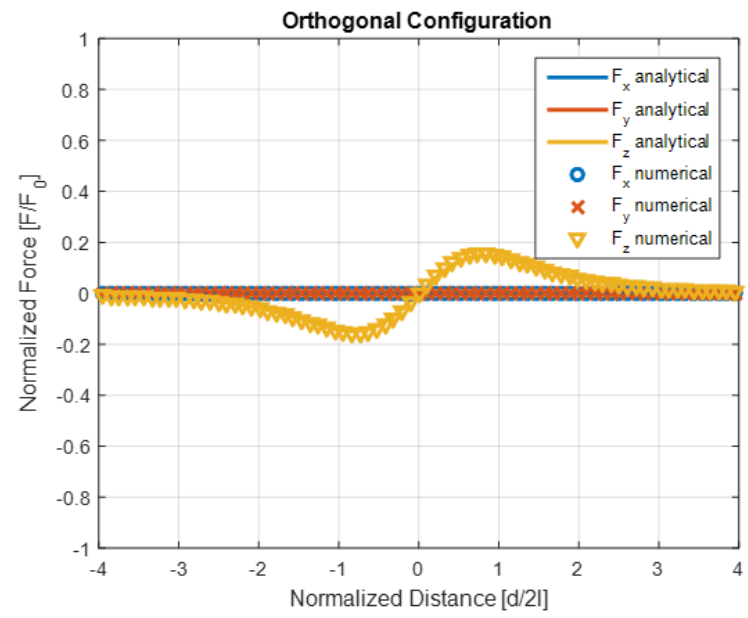

(c)

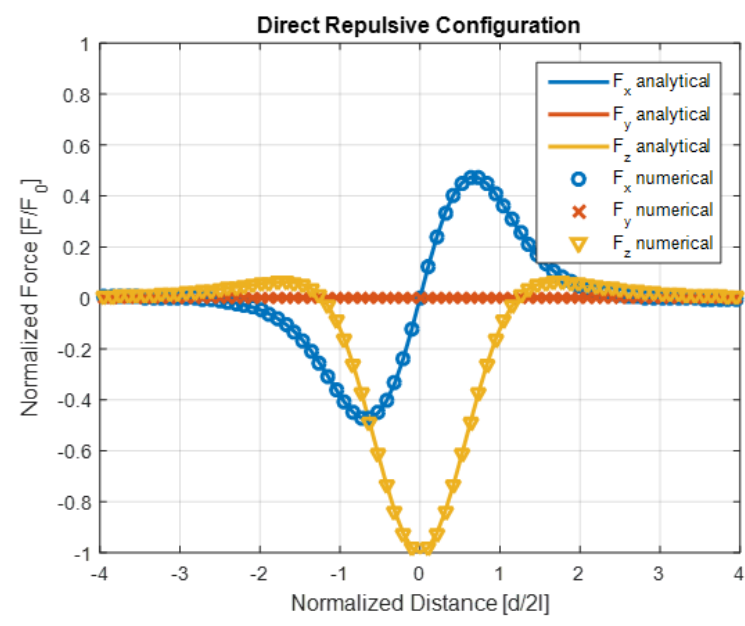

(b)

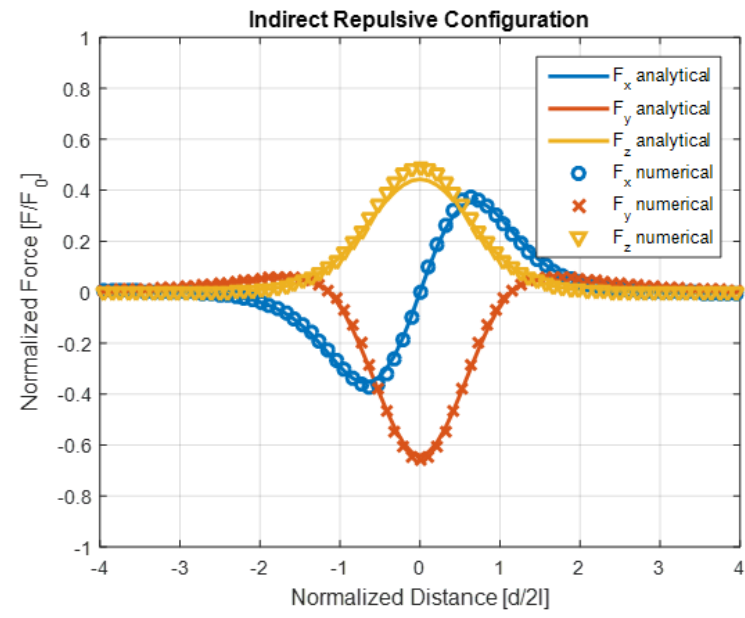

(d)

FIG. 4. Static force profiles for different magnetic plucking configurations. The transverse force is $F_{x}$ in (a) in-plane plucking configuration, and $F_{z}$ in (b),(c),(d) out-of-plane plucking configurations.

As is evident from, the analytical model matches the numerical results well. In general, the transverse force (along the direction of beam deflection) and the axial force (along the direction of beam length) are of interest for plucking. The transverse force directly deflects the beam whereas the axial force softens the beam and provides a bending moment when the beam is already deflected. Note that due to the different orientations in FIG. 2, the transverse force in the in-plane plucking configuration is $F_{x}$ whereas in the out-of-plane plucking configurations it is $F_{z}$. Although the lateral force could potentially produce a torsional movement, we leave it out in the later modelling due to a relatively high torsional rigidity of the beam applied in our study. 


\subsection{In-plane plucking}

As shown in (a), the directionality of the transverse force $F_{x}$ changes at the zero displacement point in the inplane plucking configuration. As mentioned earlier a jump phenomenon will occur due to the magnetic interaction and the cantilever restoring force. To investigate this phenomenon we formulate the following equation to find the equilibrium beam tip position with a spring model for the beam and a published dipole model[24] for the magnet for simplicity.

$$
\frac{3 \mu}{4 \pi|\mathbf{d}|^{5}}\left[\left(\mathbf{m}_{\mathbf{1}} \cdot \mathbf{d}\right) \mathbf{m}_{2}+\left(\mathbf{m}_{\mathbf{2}} \cdot \mathbf{d}\right) \mathbf{m}_{\mathbf{1}}+\left(\mathbf{m}_{\mathbf{1}} \cdot \mathbf{m}_{2}\right) \mathbf{d}-\frac{5\left(\mathbf{m}_{\mathbf{1}} \cdot \mathbf{d}\right)\left(\mathbf{m}_{2} \cdot \mathbf{d}\right)}{|\mathbf{d}|^{2}} \mathbf{d}\right] \cdot \hat{\mathbf{i}}-k x_{b}=0
$$

where

$$
\begin{gathered}
\mathbf{d}=\left[\begin{array}{cc}
-x_{m}+x_{b} & 0
\end{array}\right] \\
\hat{\mathbf{\imath}}=\left[\begin{array}{ll}
1 & 0
\end{array}\right]^{T}
\end{gathered}
$$

where $\mathbf{m}_{1}$ and $\mathbf{m}_{\mathbf{2}}$ are the dipole moments, $k$ is the equivalent beam stiffness, $x_{m}$ is the moving magnet displacement, and $x_{b}$ is the beam tip displacement. The zeros of equation (10) are plotted in FIG. 5 with arrows marking the jump locations. This bifurcation guarantees a dynamic ring down of the beam at its natural frequency after it has been deflected and released. In general the plucking occurs as long as the moving magnet passes the beam and the jump location. Thus, the jump phenomenon has little dependency on the velocity. The jump location will not move much as long as the velocity is moderate. 


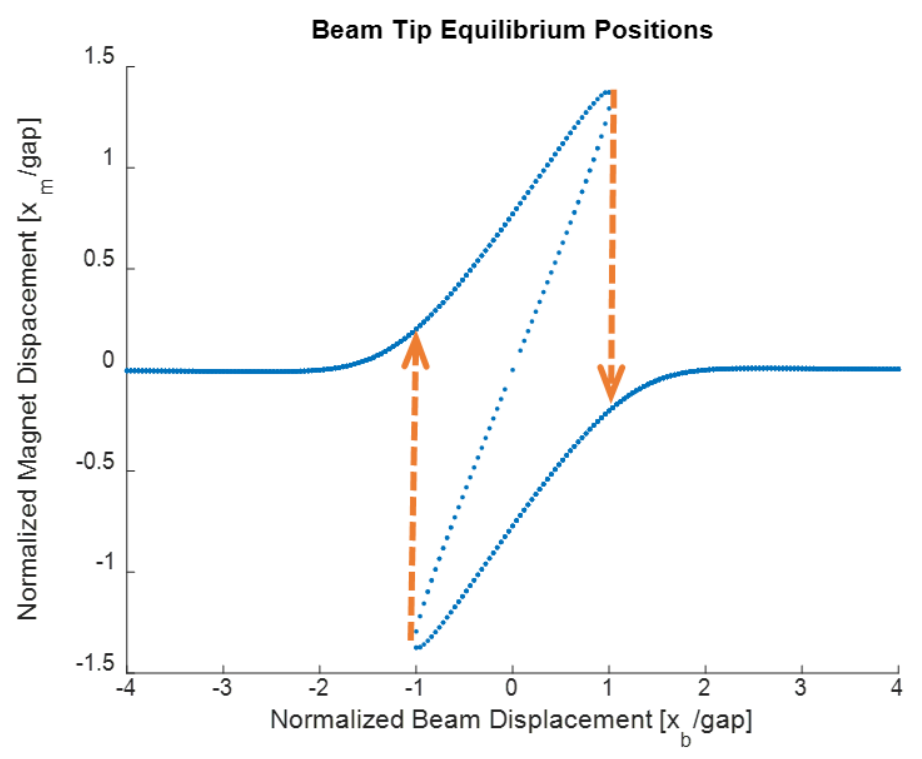

FIG. 5. Bifurcation in the in-plane plucking configuration

\subsection{Out-of-plane plucking}

For the out-of-plane plucking configurations, the transverse force $F_{z}$ is predominantly in the same direction (i.e. it does not change sign) for both the direct repulsive and the indirect repulsive configurations. While the transverse force does change its directionality in the orthogonal configuration, its magnitude is relatively small compared to the in-plane plucking configuration given the same gap between magnets. Most importantly, the orientation of the beam eliminates the jump phenomenon in out-of-plane configurations. Therefore, for out-of-plane configurations, plucking (i.e. a dynamic ring down after the beam is deflected and released at its natural frequency) occurs only when the velocity of moving magnet exceeds a certain threshold. To be more precise, it requires the force ramp to be faster than the beam can respond due to its resonant frequency. Otherwise the beam will only undergo a gradual deflection without any dynamic ring down. The voltage output is significantly reduced in this scenario. Nevertheless, the appeal of out-of-plane plucking is that we can easily embed multiple beams to increase total power output by fabricating them on a single substrate as mentioned earlier.

Among the three out-of-plane configurations, for a given gap between magnets, the direct repulsive configuration exhibits the largest transverse force difference, i.e., the absolute difference between the maximum and the minimum transverse force during actuation. The indirect repulsive configuration benefits from a large axial force whereas the orthogonal configuration operates in a pure deflecting mode, i.e., only the transverse force is generated. Although the direct repulsive configuration seems the most promising to trigger plucking, it requires a 
larger gap between magnets to avoid the pull-in effect (i.e., opposing magnets get stuck together in a side by side orientation) and a larger space for beam deflection. Since the magnets are aligned in the direction of device

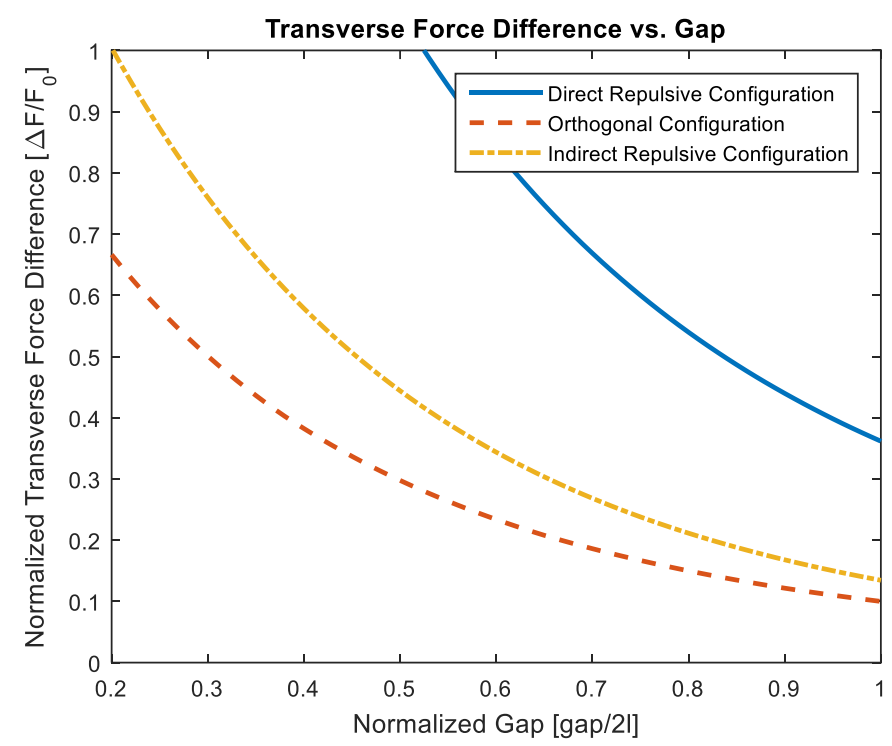

FIG. 6. Comparison of transverse force difference for different out-of-plane magnetic plucking configurations thickness, this hurts the overall power density, which is important as wearables are extremely size-sensitive. In the other two configurations magnets are highly unlikely to touch each other, thus we are able to implement a smaller gap between magnets which results in a larger force. As illustrated in FIG. 6, by implementing a smaller gap, the indirect repulsive or the orthogonal configuration can achieve a higher transverse force difference than the direct repulsive configuration with a larger gap.

In addition, the offset in the indirect repulsive configuration can be optimized to increase the transverse force. Because of the existence of a transcendental function in Eq. (3-5), a geometric scaling effect on the force between magnets cannot be easily obtained via an analytical approach by non-dimensionalization. Nevertheless, a scaling of the magnetic force $F \propto l^{2}$ does hold in some particular cases, such as the contact force, when two magnets are aligned precisely[19]. Although no global geometric scaling invariance appears in the equation, we can numerically demonstrate the optimal range of operation for the indirect repulsive configuration. FIG. 7 shows that for a given normalized gap there is an optimal normalized offset to achieve the maximum transverse force regardless of the magnet length. In this case the magnet length is varied from $1 \mu \mathrm{m}$ to $1 \mathrm{~mm}$. The optimal offset marked by the black arrow in Fig. 6 is dependent on the gap between magnets and will increase as the gap grows. 


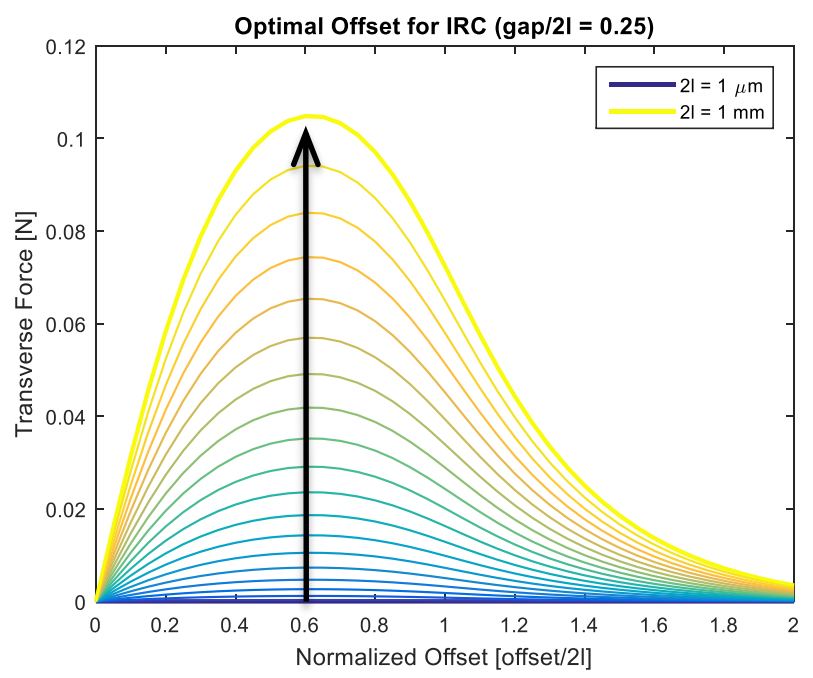

(a)

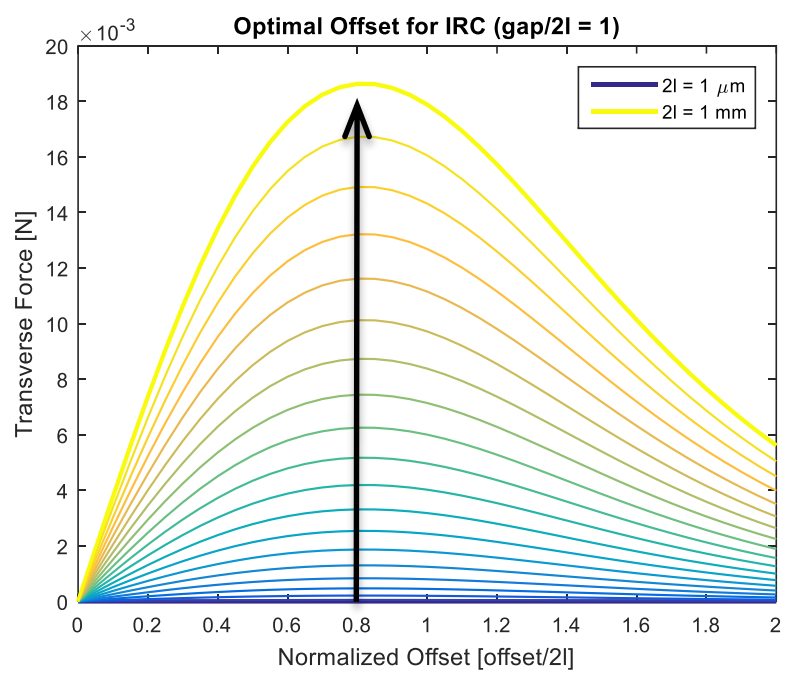

(b)

FIG. 7. Optimal offset for indirect repulsive configuration with the magnet length $2 l$ varied from $1 \mu \mathrm{m}$ to $1 \mathrm{~mm}$ (marked by the arrow): (a) normalized optimal offset is around 0.6 when the normalized gap is 0.25 , (b) normalized optimal offset is around 0.8 when the normalized gap is 1.

\section{A model for magnetically plucked piezoelectric beams}

A cantilevered composite beam, as illustrated in FIG. 8, is a common implementation of a piezoelectric energy harvester. Numerous models of a piezoelectric beam, typically under harmonic base excitation, have been published. In general, there are two approaches: the lumped parameter model and the distributed parameter model. A detailed review of these various modelling methods is given by Erturk and Inman[25]. For a magnetically plucked piezoelectric beam, however, the forcing mechanism is the magnetic force acting at the free end instead of the base excitation. Previous modelling attempts[21,26] tend to simplify the magnetic force model as in those cases the permanent magnets are in a direct repulsive or attractive configuration. However, when considering more complex magnet orientations, these simplified models lack accuracy. 


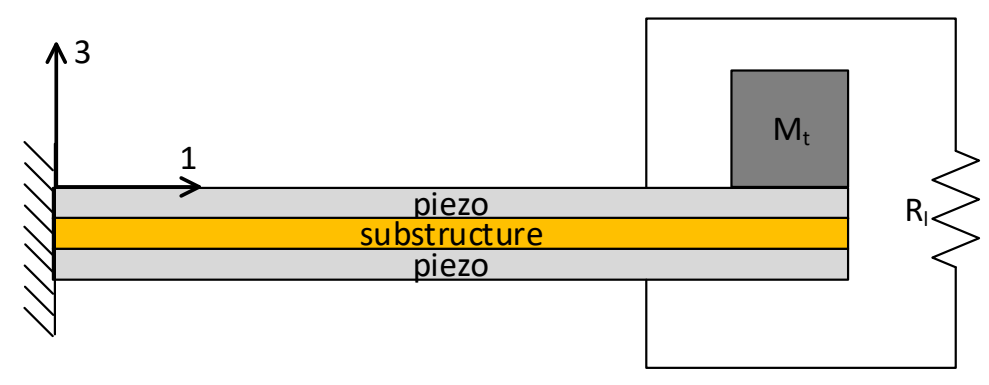

FIG. 8. Piezoelectric bimorph beam with magnet attached to the free end in series connection

Our modeling procedure follows Erturk and Inman's distributed parameter piezoelectric beam model[27] with modified forcing conditions and the addition of magnetic coupling. The piezoelectric bimorph is connected in series. Based on Euler-Bernoulli beam theory, the kinetic and potential energy of a cantilever bimorph beam with active length $L$ and width $b$ subjected to a transverse force $F_{z}$ and an axial force $F_{y}$ is given by

$$
\begin{gathered}
T=\frac{1}{2} \int_{0}^{L} m\left[\frac{\partial w(x, t)}{\partial t}\right]^{2} d x+\frac{1}{2} M_{t}\left[\frac{\partial w(x, t)}{\partial t}\right]_{x=L}^{2}+\frac{1}{2} I_{t}\left[\frac{\partial^{2} w(x, t)}{\partial x \partial t}\right]_{x=L}^{2} \\
U=\frac{1}{2} \int_{0}^{L}\left[Y I\left[\frac{\partial^{2} w(x, t)}{\partial x^{2}}\right]^{2}-\vartheta_{s} v(t) \frac{\partial^{2} w(x, t)}{\partial x^{2}}\right] d x
\end{gathered}
$$

where

$$
\vartheta_{S}=b \int_{\frac{1}{2} h_{S}}^{\frac{1}{2} h_{S}+h_{p}} \frac{d_{31} \bar{c}_{11}^{E}}{h_{p}} z d z=\frac{1}{2} d_{31} \bar{c}_{11}^{E} b\left(h_{p}+h_{S}\right)
$$

where $w(x, t)$ is the transverse displacement of the beam, $v(t)$ is the voltage across the two piezoelectric layers, $M_{t}$ and $I_{t}$ are the mass and the mass moment of inertia of the proof mass (i.e., a permanent magnet) at the free end, and $d_{31}$ is the piezoelectric strain coefficient. $Y I$ is the effective bending stiffness of the composite beam, which can be expressed as a function of its piezoelectric layer and substructure layer thickness $h_{p}$ and $h_{s}$ with the corresponding elastic modulus $\bar{c}_{11}^{E}$ and $Y_{S}$ respectively:

$$
Y I=\frac{2 b}{3}\left\{Y_{s} \frac{h_{s}^{3}}{8}+\bar{c}_{11}^{E}\left[\left(h_{p}+\frac{h_{s}}{2}\right)^{3}-\frac{h_{s}^{3}}{8}\right]\right\}
$$


$m$ is the mass per unit length of the beam determined by the beam geometry and the densities of the piezoelectric and substructure layers $\rho_{p}$ and $\rho_{s}$ :

$$
m=b\left(\rho_{s} h_{s}+2 \rho_{p} h_{p}\right)
$$

The extended Hamilton's principle can be applied in the absence of mechanical damping effects:

$$
\delta \int_{t_{1}}^{t_{2}}\left(T-U+W_{i e}+W_{n c}\right) d t=0
$$

where $W_{n c}$ is the total work done by the non-conservative mechanical forces including the transverse force $F_{z}$, the axial force $F_{y}$ and the work due to the non-conservative electric charge output $Q(t)$, and $W_{i e}$ is the internal electrical energy in the piezoelectric layers given by

$$
\begin{gathered}
W_{n c}=-\int_{0}^{L} F_{y}\left[\frac{\partial w(x, t)}{\partial x}\right]^{2}+F_{z} w(L, t)+Q(t) v(t) \\
W_{i e}=\frac{1}{2}\left[\int_{0}^{L} \vartheta_{s} v(t) \frac{\partial^{2} w(x, t)}{\partial x^{2}} d x+\frac{1}{2} C_{p} v^{2}(t)\right]
\end{gathered}
$$

Here the effective capacitance of the piezoelectric layer $C_{p}$ is a function of $\bar{\epsilon}_{33}^{S}$, the material permittivity evaluated at constant strain and beam geometry. Note that the total beam length, $L_{t}$, is used here instead of the active length $L$ as the electrode covers the entire beam surface. In practice the capacitance can be simply obtained from measurement.

$$
C_{p}=\frac{\bar{\epsilon}_{33}^{S} b L_{t}}{h_{p}}
$$

The governing equations of the system can be obtained from the electromechanical Lagrange's equations based on the extended Hamilton's principle. By introducing viscous air damping and strain rate damping back into the system, the equation of motion can be expressed as 


$$
\begin{aligned}
& Y I \frac{\partial^{4} w(x, t)}{\partial x^{4}}+c_{S} I \frac{\partial^{5} w(x, t)}{\partial x^{4} \partial t}+c_{a} \frac{\partial w(x, t)}{\partial t}+m \frac{\partial^{2} w(x, t)}{\partial t^{2}}+\left[M_{t} \frac{\partial^{2} w(x, t)}{\partial t^{2}}+I_{t} \frac{\partial^{3} w(x, t)}{\partial x \partial t^{2}}\right] \delta(x-L)-F_{y}(t) \frac{\partial^{2} w(x, t)}{\partial x^{2}}- \\
& \vartheta_{s} v(t)\left[\frac{d \delta(x)}{d x}-\frac{d \delta(x-L)}{d x}\right]=F_{z}(t) \delta(x-L)
\end{aligned}
$$

where $\delta(x)$ is the Dirac delta function, $c_{s}$ and $c_{a}$ are the strain rate and viscous air damping coefficients respectively. These coefficients can be calculated from the first two modal damping coefficients[27], which are typically obtained experimentally using the logarithmic decrement method. We follow the standard assumed-modes method to represent the displacement of the beam $w(x, t)$ as a convergent series of the eigenfunctions:

$$
w(x, t)=\sum_{r=1}^{\infty} \phi_{r}(x) \eta_{r}(t)
$$

where $\phi_{r}(x)$ and $\eta_{r}(t)$ are the mass normalized eigenfucntion and the modal mechanical coordinate of the cantilever beam with respect to its $r$ th mode shape. The equation of motion can be further reduced to the modal coordinate by substituting Eq. (23) into Eq. (22) and integrating over the beam length:

$$
\frac{d^{2} \eta_{r}(t)}{d t^{2}}+2 \zeta_{r} \omega_{r} \frac{d \eta_{r}(t)}{d t}+\omega_{r}^{2} \eta_{r}(t)-\vartheta_{s}\left[\frac{d \phi_{r}(x)}{d x}\right]_{x=L} v(t)=\left[F_{z}(t) \phi_{r}(x)\right]_{x=L}
$$

In the above equation, $\zeta_{r}$ is the modal damping ratio and $\omega_{r}$ is the effective undamped modal frequency of the beam in the presence of the axial excitation force. The corresponding electrical equation of a bimorph piezoelectric beam in series connection with a resistive load $R_{l}$ is

$$
\frac{C_{p}}{2} \frac{d v(t)}{d t}+\frac{v(t)}{R_{l}}+\sum_{r=1}^{n} \vartheta_{S}\left[\frac{d \phi_{r}(x)}{d x}\right]_{x=L} \frac{d \eta_{r}(t)}{d t}=0
$$

We can obtain the full magnetically plucked piezoelectric beam model by combining the piezoelectric beam model and the magnetic force model. Eq. (2) shows that each magnetic force component is a sum of 64 values of the function $\psi$, which could be computationally expensive in a dynamic simulation where the force needs to be updated at each time step. Thus, we desire a further simplification without much sacrifice in precision for a full energy harvester model. Since the deflection of the beam adds another dimension into the dynamic force profile, 
we applied a surface fit to the analytical solution using a function as the product of a Gaussian and a polynomial in two dimensions $x$ and $z$ respectively:

$$
F=a\left(\left(z-z_{0}\right)+\left(z-z_{1}\right)^{2}\right) \exp \left(\frac{-\left(x-x_{0}\right)^{2}}{\sigma^{2}}\right)
$$

where $a, x_{0}, z_{0}, z_{1}$, and $\sigma$ are fitting parameters.

FIG. 9 gives an example of the fitted surface for the transverse magnetic force in the indirect repulsive configuration as the function of the rotor angle and the beam displacement. A similar procedure can be applied to other configurations.

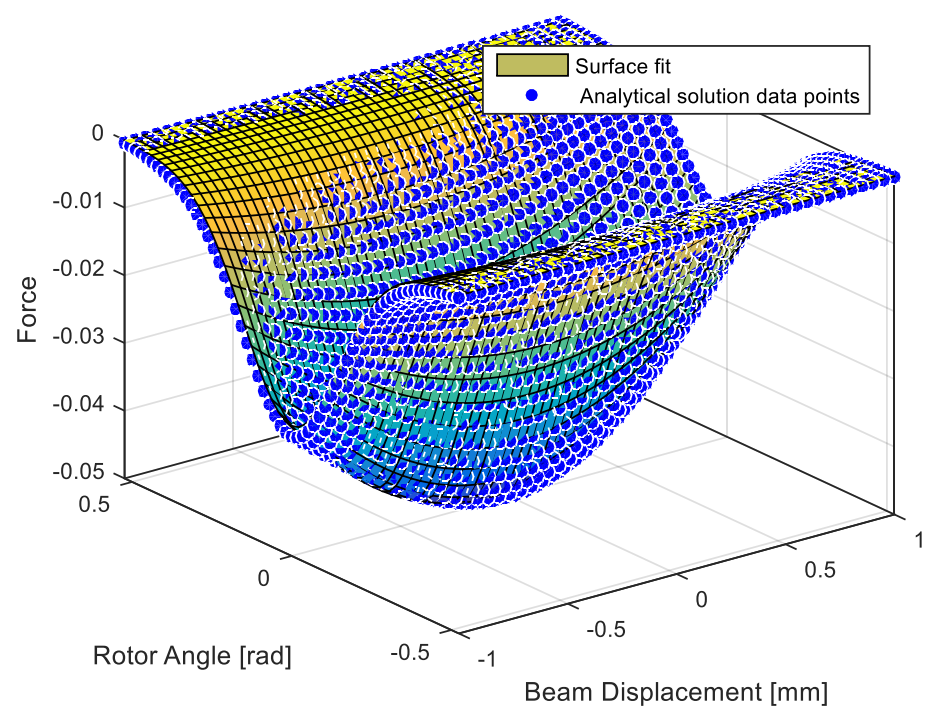

FIG. 9. Surface fitting for the transverse force $F_{z}$ in indirect repulsive configuration

\section{Experimental results}

\subsection{Experimental setup}

To validate the magnetically plucked piezoelectric beam model we designed an experimental set-up as shown in FIG. 10. A piezoelectric beam made by Piezo Systems Inc. is clamped on an adjustable XYZ stage with alternative clamps for different magnetic plucking configurations. The brass reinforced piezoelectric beam is made with PSI-5A4E piezoceramic material and is coated with electrodes on each side. The original length of the beam is $31.4 \mathrm{~mm}$ and is shortened to an active length of $26.4 \mathrm{~mm}$ due to the clamp and the tip magnet. The complete list of parameters of the beam is given in Table 1 . 
The excitation is achieved with a motor-driven aluminum swing arm. The motor driving profile can be varied to achieve different magnetic plucking velocities. N50 cube magnets $(2 \mathrm{~mm} \times 2 \mathrm{~mm} \times 2 \mathrm{~mm})$ are attached at both the end of the piezoelectric beam and the swing arm with orientations corresponding to each magnetic plucking configuration. The effective swing arm length for the magnet is $62.5 \mathrm{~mm}$, which results in a tangential velocity of $196 \mathrm{~mm} \mathrm{~s}^{-1}$ at $\pi \mathrm{rad} \mathrm{s}^{-1}$. During the excitation, we measure the voltage output from the electrodes across a $120 \mathrm{k} \Omega$ resistor based on impedance matching.

Table 1. Parameters for the piezoelectric bimorph beam

\begin{tabular}{ll}
\hline \hline Parameter & Value \\
\hline$L$ & $26.4 \mathrm{~mm}$ \\
$b$ & $3.2 \mathrm{~mm}$ \\
$h_{s}$ & $0.1 \mathrm{~mm}$ \\
$h_{p}$ & $0.14 \mathrm{~mm}$ \\
$\rho_{s}$ & $7800 \mathrm{~kg} \mathrm{~m}^{-3}$ \\
$\rho_{p}$ & $8500 \mathrm{~kg} \mathrm{~m}^{-3}$ \\
$Y_{S}$ & $100 \times 10^{9} \mathrm{~N} \mathrm{~m}^{-2}$ \\
$\bar{c}_{11}^{E}$ & $66 \times 10^{9} \mathrm{~N} \mathrm{~m}^{-2}$ \\
$d_{31}$ & $-190 \times 10^{-12} \mathrm{~m} \mathrm{~V}^{-1}$ \\
$\bar{\epsilon}_{33}^{S}$ & $1.593 \times 10^{-8} \mathrm{~F} \mathrm{~m}^{-1}$ \\
$C_{p}$ & $10 \mathrm{nF}$ \\
$R_{l}$ & $120 \mathrm{k} \Omega$ \\
\hline \hline
\end{tabular}

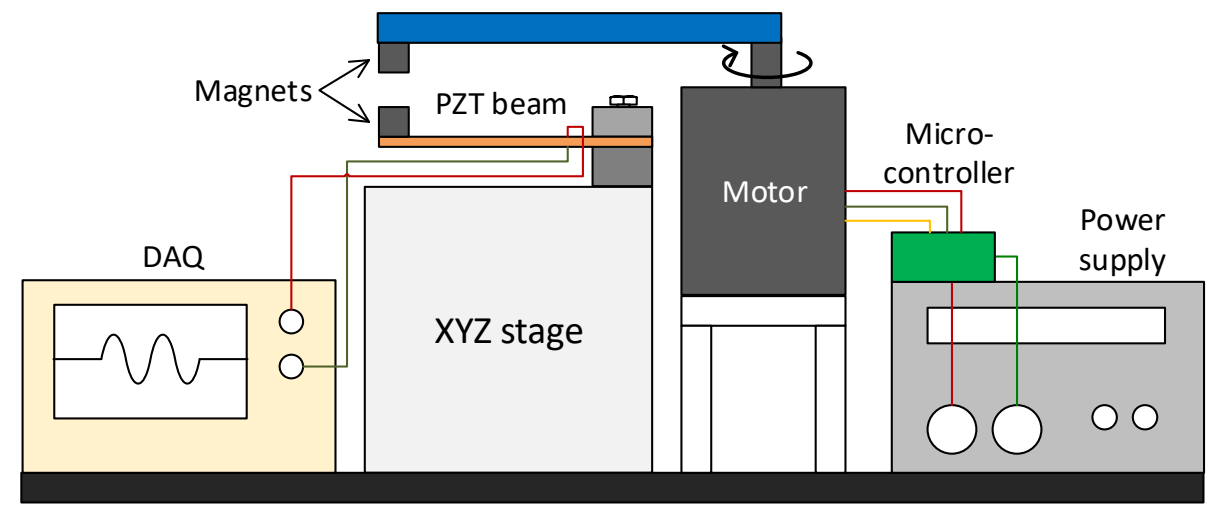

FIG. 10. Experimental set-up for the magnetically plucked piezoelectric beam model with different magnetic plucking configurations

\subsection{Results and discussion}

In this section we present time domain voltage waveforms from both simulation and experimental results for different magnetic plucking configurations. The simulation was conducted in Matlab. Again, within the small region 
of magnetic interaction, the path of the moving magnet is approximated as linear and thus the small angles between the opposing surfaces of the magnets are neglected. In general, a good match in terms of waveform shape between simulation and measurement is demonstrated with a slight discrepancy in the value of voltage output. This is most likely due to inexactness in material properties of the piezoelectric beam and the residual flux density of the permanent magnet as in most cases the material properties are given as a range. Inaccuracies in adjusting the gap and the offset between magnets also contribute to the overall error.

For the in-plane plucking configuration, the main takeaway from the previous analysis is that the plucking is due to the bifurcation in the resultant forces, and thus places no requirement on the moving magnet velocity. This is illustrated in FIG. 11 with motor driving speed of $\pi / 2 \mathrm{rad} \mathrm{s}^{-1}$ and $2 \pi \mathrm{rad} \mathrm{s}^{-1}$. The maximum voltage output during the initial deflection is around $50 \mathrm{~V}$ for both cases. A clear ring down of oscillations is demonstrated, indicating a clean release of the beam. In this case, a higher excitation velocity will increase the number of excitations per unit time but the energy produced per excitation improves only marginally.
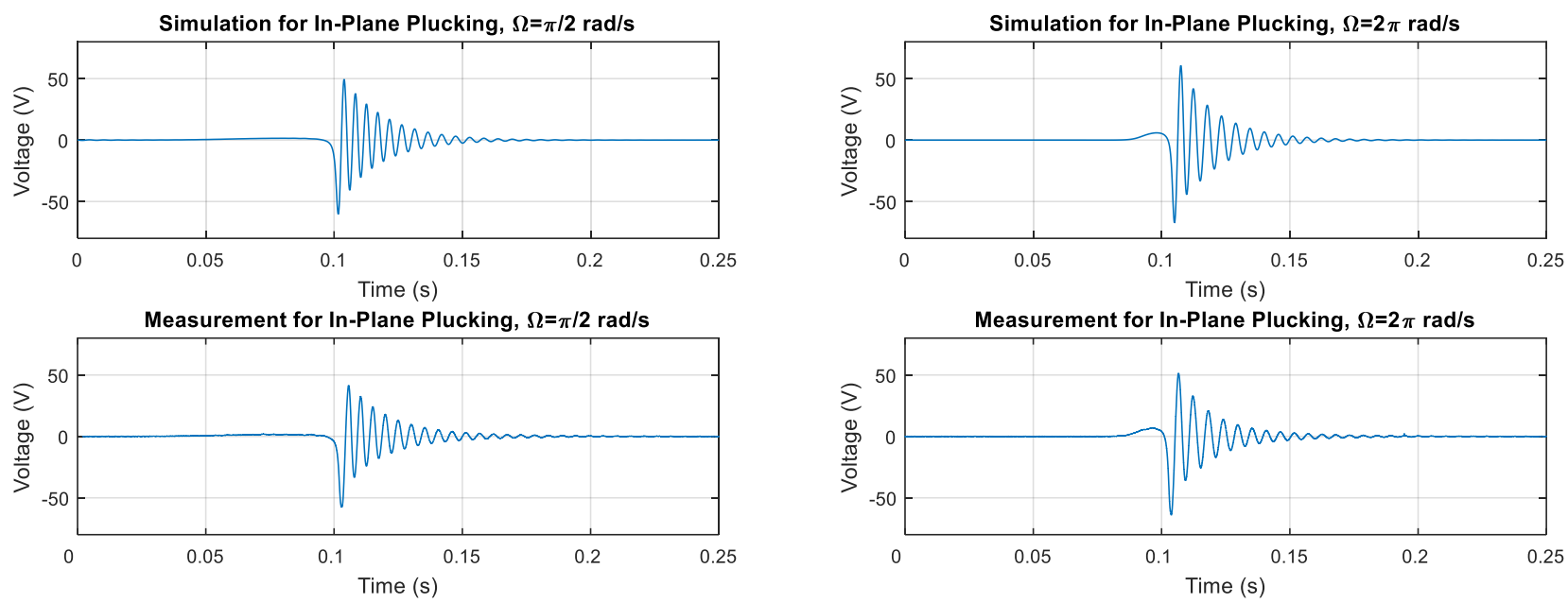

(a)

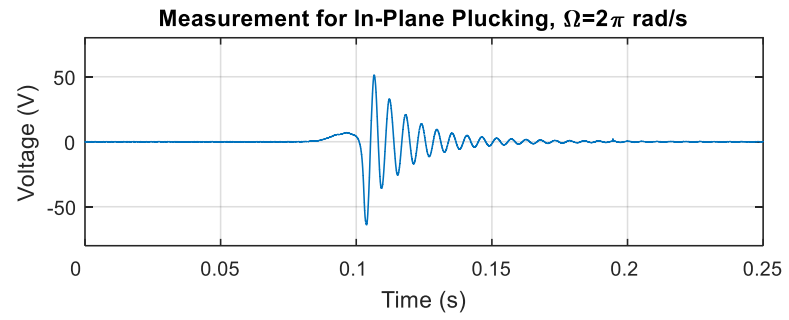

(b)

FIG. 11. Simulated and measured voltage output from the piezoelectric beam for the in-plane plucking configuration $(\mathrm{gap}=$ $1 \mathrm{~mm}$ ) with motor driving speed of (a) $\pi / 2 \mathrm{rad} \mathrm{s}^{-1}$ and (b) $2 \pi \mathrm{rad} \mathrm{s}^{-1}$

For out-of-plane plucking configurations, as shown in FIG. 12 to FIG. 13, the dynamics of the piezoelectric beam are dependent on the velocity of the moving magnet. A higher driving speed will produce a cleaner ring down and a higher voltage. At a lower motor driving speed of $\pi \operatorname{rad~s}^{-1}$, the beam in the direct repulsive and indirect repulsive configuration only exhibits a gradual deflection with minimal ring down whereas a biased ring down 
exists in the orthogonal configuration. At a higher motor driving speed of $2 \pi \mathrm{rad} \mathrm{s}^{-1}$, the voltage approximately doubles with a cleaner ring down after the initial deflection for all out-of-plane plucking configurations. However, there is a big voltage reduction between the first and the second oscillation, indicating a reluctant release from the magnetic force. The voltage reduction is much larger in the direct repulsive and indirect repulsive configuration than in the orthogonal configuration. This is because in the orthogonal configuration the magnetic force directionality changes which guarantees a positive to negative deflection. Magnetic coupling exists in the entire first oscillation of the beam for all configurations, however in the in-plane plucking configuration the beam undergoes a true free oscillation after the initial deflection. This is further illustrated in FIG. 15 with the simulated beam tip displacement for each configuration.
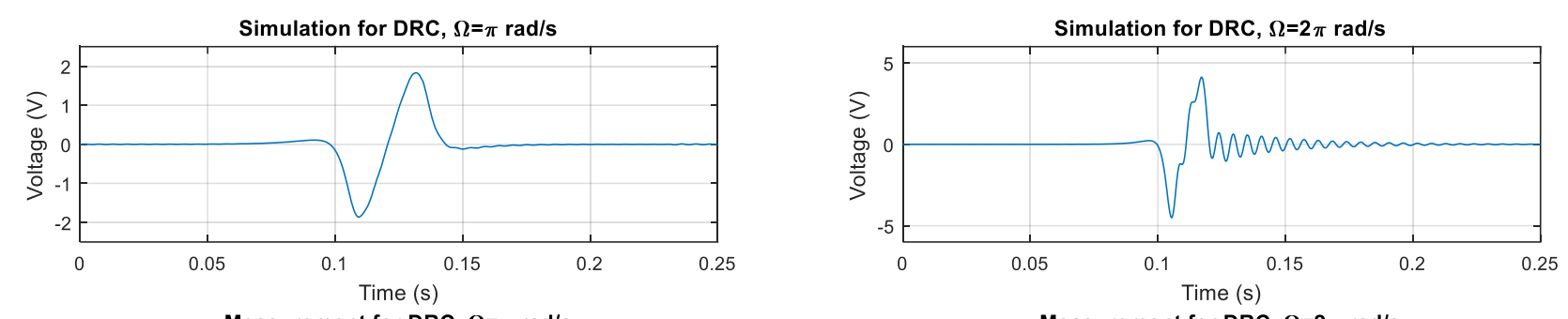

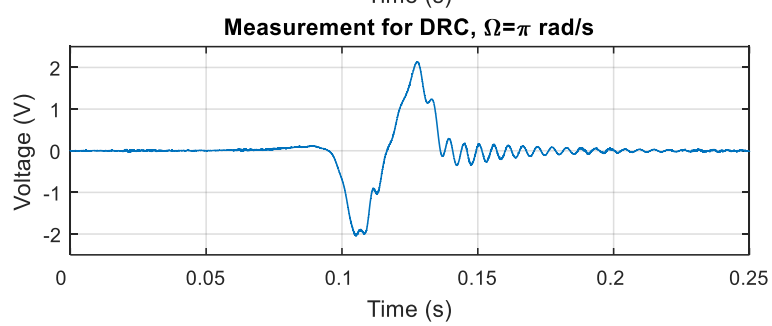

(a)

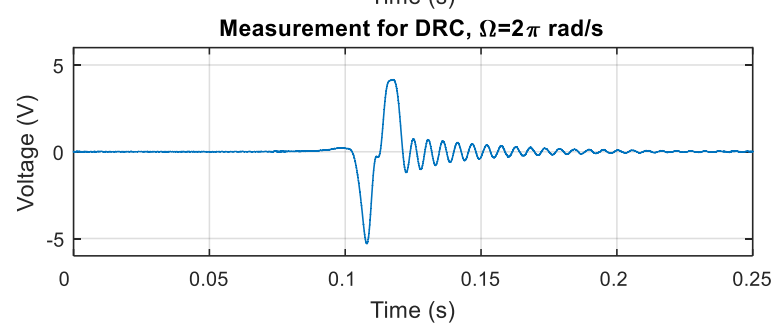

(b)

FIG. 12. Simulated and measured voltage output from the piezoelectric beam for the direct repulsive configuration (gap $=2$ $\mathrm{mm}$ ) with motor driving speed of (a) $\pi \mathrm{rad} \mathrm{s}^{-1}$ and (b) $2 \pi \mathrm{rad} \mathrm{s}^{-1}$

Note that the gap and the offset between magnets used in the experiment are different from the earlier static force profile. We applied a $2 \mathrm{~mm}$ gap in the direct repulsive configuration to avoid the pull-in effect, which results in a lower voltage due to the weak magnetic coupling whereas we can implement a $0.5 \mathrm{~mm}$ gap to increase the magnetic coupling for the indirect repulsive and orthogonal configurations. A $1 \mathrm{~mm}$ offset is applied in the indirect repulsive configuration, which is close to its optimal value. 

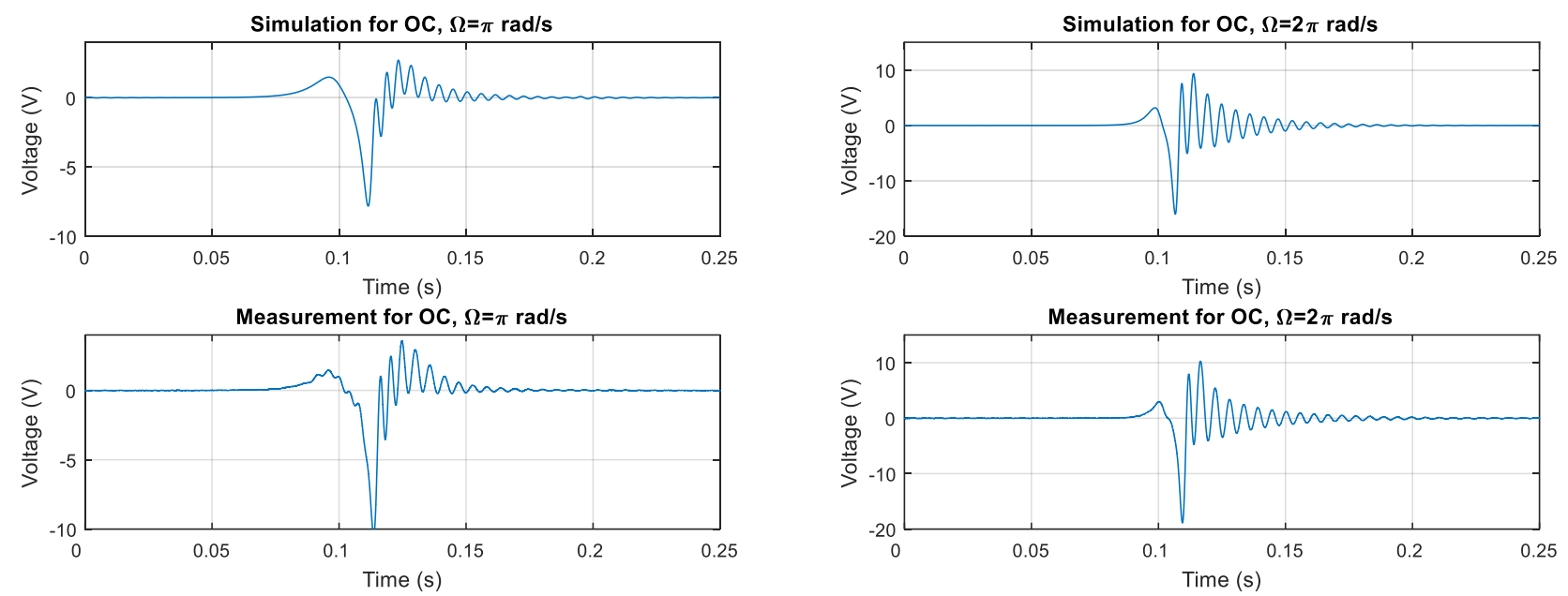

(a)

(b)

FIG. 14. Simulated and measured voltage output from the piezoelectric beam for the orthogonal configuration $(\mathrm{gap}=0.5 \mathrm{~mm})$ with motor driving speed of (a) $\pi \mathrm{rad} \mathrm{s}^{-1}$ and (b) $2 \pi \mathrm{rad} \mathrm{s}^{-1}$
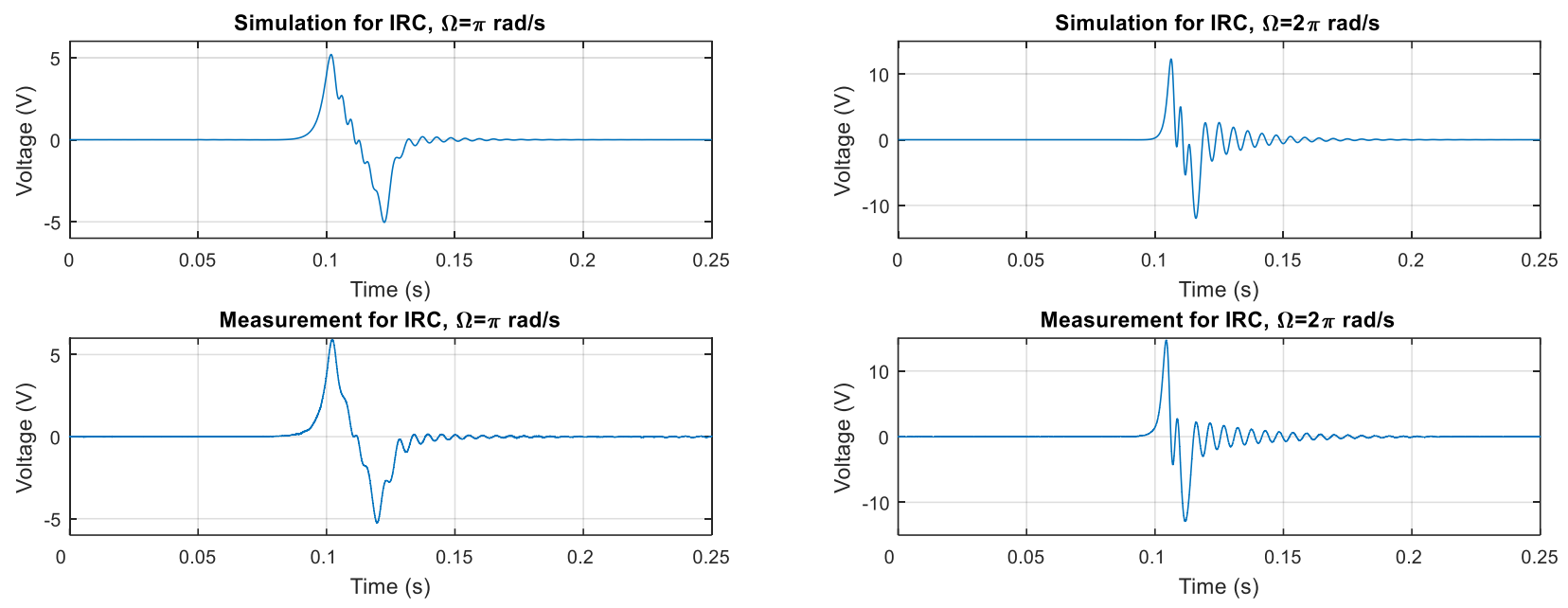

(a)

(b)

FIG. 13. Simulated and measured voltage output from the piezoelectric beam for the indirect repulsive configuration $(\mathrm{gap}=$ $0.5 \mathrm{~mm}$ ) with motor driving speed of (a) $\pi \mathrm{rad} \mathrm{s}^{-1}$ and (b) $2 \pi \mathrm{rad} \mathrm{s}^{-1}$

In general, the in-plane plucking configuration produces more power than the out-of-plane plucking configurations given the same conditions at low driving speeds due to its larger initial deflection and the capability of free oscillation. In practice, however, the in-plane configuration requires a larger driving torque to push the beam through the bifurcation point. For a displacement-driven excitation where the driving force or torque is sufficiently large such as the knee-joint motion [11], the in-plane plucking configuration is the obvious choice. For inertiadriven excitations, when the amplitude of acceleration is low, the in-plane plucking configuration might not function as the moving magnet may not push through the beam. In this case, although the out-of-plane plucking 
configurations generate less energy per pluck, they may be preferable. A fundamental difference between the two lies in its dependency on velocity to extract energy from the system. The out-of-plane configurations is evidently more sensitive to the velocity of the moving magnet.

We choose to use the maximum power output instead of efficiency to compare different configurations in terms of performance. The efficiency is used typically on the transducer level. It can be defined as the ratio of energy output to potential magnetic energy at its maximum in this case. However it leaves out the velocity of moving magnets which is vital in the dynamic study. Thus a direct comparison of output power is more suitable.

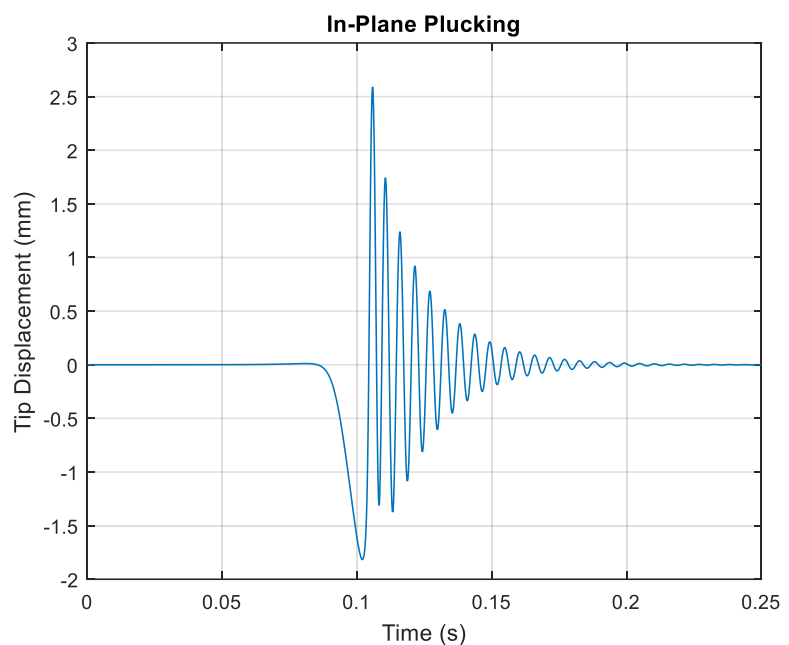

(a)

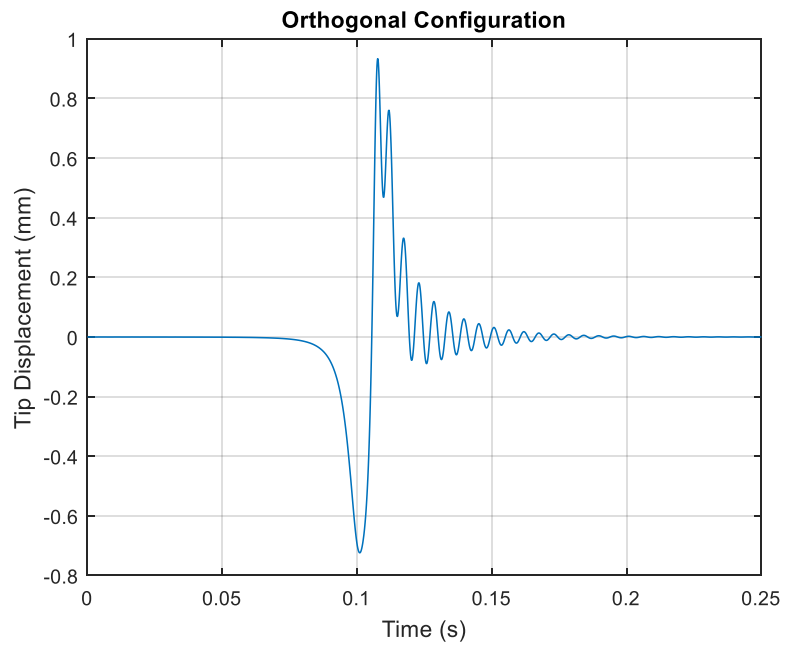

(c)

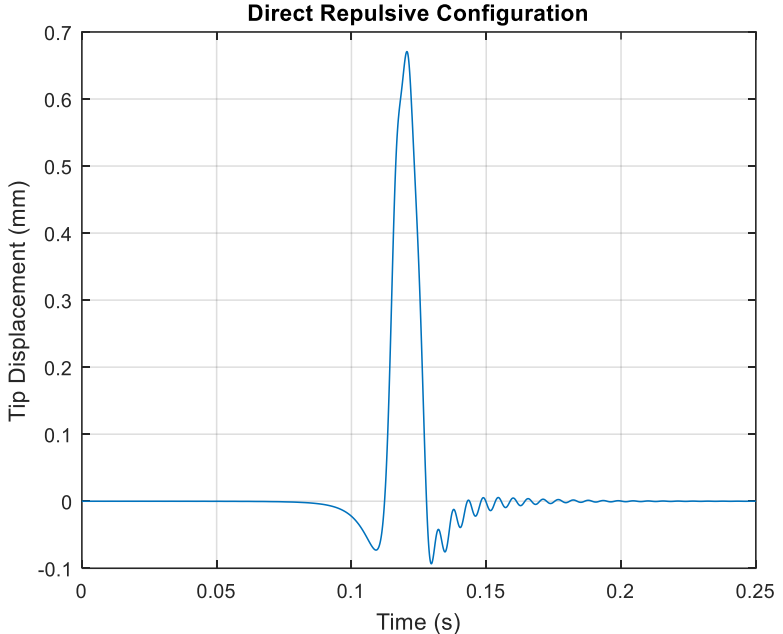

(b)

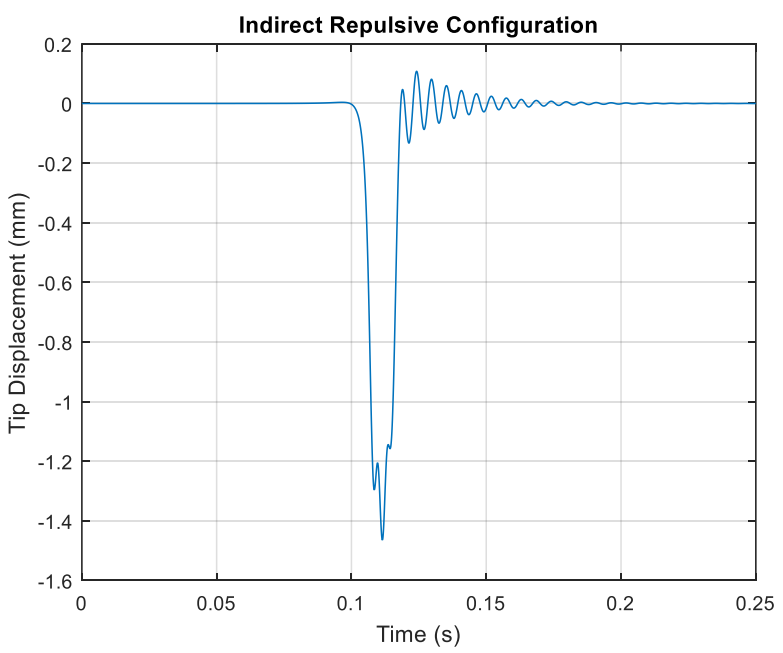

(d)

FIG. 15. Simulated beam tip displacement for different magnetic plucking configurations: (a) in-plane plucking configuration, (b) direct repulsive configuration, (c) orthogonal configuration, and (d) indirect repulsive configuration 
To gain insight into the scaling relationship between the excitation frequency (i.e., the motor driving frequency) and the power output, we extend the simulation into a larger frequency range to compare in-plane plucking and outof-plane plucking. We choose the indirect repulsive configuration as the representative configuration. The simulation is built upon the assumption that the piezoelectric beam is actuated twice per motor cycle which is different from the test set-up. FIG. 16 shows both RMS power and maximum voltage as a function of motor driving frequency. For the in-plane plucking configuration, the maximum voltage scales approximately linearly with the driving frequency until a certain point around $5 \mathrm{~Hz}$. Beyond this speed, the beam has not completely rung down when it gets plucked again. Thus, as the moving magnet starts to interact with the magnet on the beam, there will be an initial deflection, which affects the maximum voltage significantly. We call this the oscillation overlay effect. The power output follows a quadratic curve initially and drops after the oscillation overlay point. Consequently, there is an optimal driving frequency to achieve the maximum power output, which means that the number of actuations per cycle (determined by the number of beams and the number of magnets) can be optimized for a welldefined rotational input. This optimal driving frequency is also dependent on the magnetic configuration and the beam resonant frequency. Given a stiffer beam the optimal point will move towards higher frequencies. The irregular data points above $5 \mathrm{~Hz}$ indicate that the oscillation overlay introduces nondeterminism into the system. For the indirect repulsive configuration, the maximum voltage grows linearly at a much larger rate with the motor driving frequency initially and goes into saturation at around $5 \mathrm{~Hz}$. The oscillation overlay has a smaller effect on the voltage output in this case. At lower frequencies the beam only exhibits a gradual deflection which, due to a constant resistive load, generates a very low voltage output. Therefore the indirect repulsive configuration generates minimal power output below $2 \mathrm{~Hz}$. An anomaly occurs at around $2.5 \mathrm{~Hz}$ due to a cancelling effect that reduces the dynamic ring down. At this critical driving speed, the magnetic force opposes the second oscillation of the beam 
which significantly affects the power output. We have only examined frequencies below $10 \mathrm{~Hz}$ as this is where frequency up-conversion usually applies.

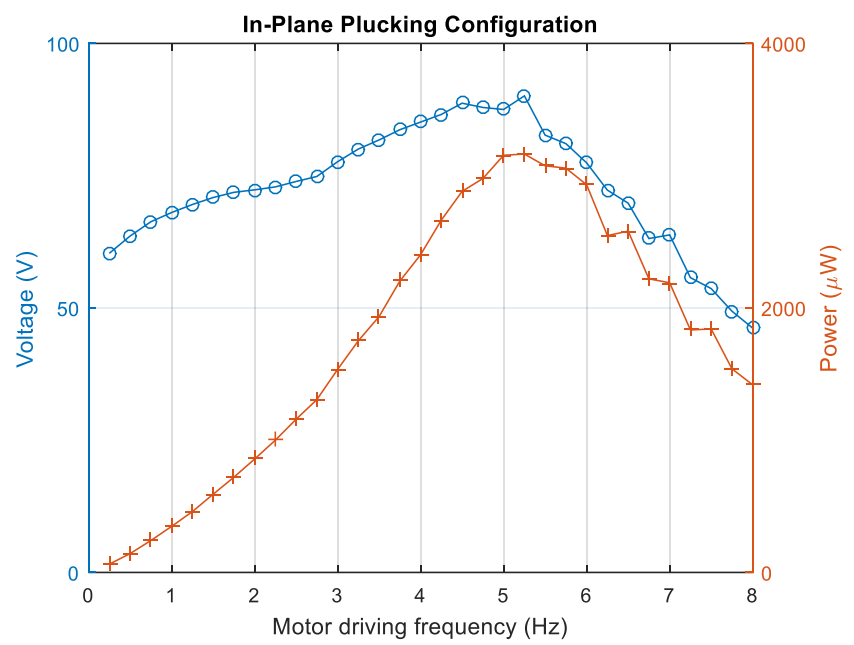

(a)

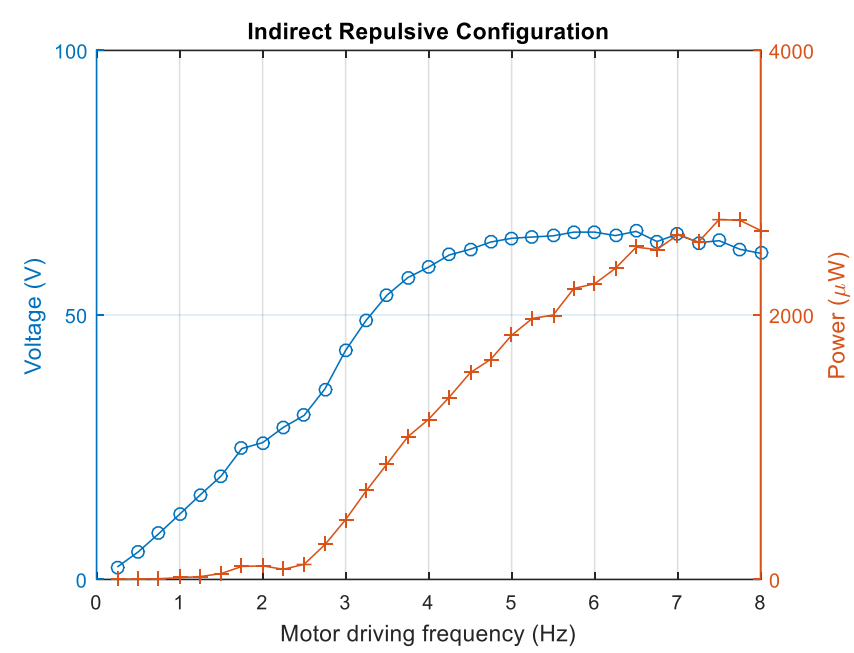

(b)

FIG. 16. Simulated maximum voltage and average power output as a function of motor driving frequency for (a) in-plane plucking configuration and (b) indirect repulsive configuration

\section{Conclusions}

This paper explores alternative configurations to achieve magnetic plucking in frequency up-converting energy harvesters. A piezoelectric beam can be plucked in or out of the plane of motion of the magnet. We presented three configurations to achieve out-of-plane plucking: the direct repulsive configuration, the orthogonal configuration, and the indirect repulsive configuration. An initial analysis based on a static force profile obtained from an analytical permanent magnet force model explains the underlying physics in achieving the magnetic plucking. Whereas the in-plane plucking configuration induces a jump phenomenon by creating a bifurcation, the out-of-plane plucking configurations rely on the speed of the moving magnet to resemble a narrow Gaussian function. The primary advantage of the out-of-plane plucking configurations is the capability of fabricating multiple piezoelectric beams on a single substrate to increase power density with lower assembly difficulty, which is especially useful in micro scale implementations.

To further study the dynamics of the magnetic plucking we derived a system level model incorporating a distributed parameter piezoelectric beam model and the analytical permanent magnet force model. A surface fitting technique can be applied to the 3 dimensional magnet force profile to reduce computation time. We developed a 
test setup to validate the model with a controlled excitation. In general, we have observed a good agreement between simulation and measurement. We extended the simulation to investigate the scaling relationship between power output and driving frequency. In-plane plucking and out-of-plane plucking exhibit different power vs. frequency profiles which indicates that a high-level optimization can be achieved by selection of the number and spacing of beams and magnets to implement an eccentric-rotor based frequency up-converting energy harvesting system.

\section{Acknowledgement}

This research is supported by National Science Foundation through the NSF Nanosystems Engineering Research Center (NERC) for Advanced Self-Powered Systems of Integrated Sensors and Technologies (ASSIST) under award number EEC 1160483. The authors would also like to thank Xiaokun Ma and Professor Chris Rahn at Pennsylvania State University for providing support on beam modeling.

\section{References}

[1] M. El-hami, P. Glynne-Jones, N.M. White, M. Hill, S. Beeby, E. James, A. D. Brown, J.N. Ross, Design and fabrication of a new vibration-based electromechanical power generator, Sensors Actuators A Phys. 92 (2001) $335-342$. doi:10.1016/S0924-4247(01)00569-6.

[2] S. Roundy, P.K. Wright, J. Rabaey, A study of low level vibrations as a power source for wireless sensor nodes, Comput. Commun. 26 (2003) 1131-1144. doi:10.1016/S0140-3664(02)00248-7.

[3] P.D. Mitcheson, T.C. Green, E.M. Yeatman, A.S. Holmes, Architectures for vibration-driven micropower generators, J. Microelectromechanical Syst. 13 (2004) 429-440. doi:10.1109/JMEMS.2004.830151.

[4] S. Roundy, J. Tola, Energy harvester for rotating environments using offset pendulum and nonlinear dynamics, Smart Mater. Struct. 23 (2014) 105004. doi:10.1088/0964-1726/23/10/105004.

[5] A. Pantelopoulos, N.G. Bourbakis, A Survey on Wearable Sensor-Based Systems for Health Monitoring and Prognosis, IEEE Trans. Syst. Man, Cybern. Part C (Applications Rev. 40 (2010) 1-12. doi:10.1109/TSMCC.2009.2032660.

[6] J.M. Donelan, Q. Li, V. Naing, J.A. Hoffer, D.J. Weber, A.D. Kuo, Biomechanical Energy Harvesting: Generating Electricity During Walking with Minimal User Effort, Science (80-. ). 319 (2008).

[7] P.D. Mitcheson, E.M. Yeatman, G.K. Rao, A.S. Holmes, T.C. Green, Energy Harvesting From Human and Machine Motion for Wireless Electronic Devices, Proc. IEEE. 96 (2008) 1457-1486.

[8] P. Pillatsch, E.M. Yeatman, A.S. Holmes, A piezoelectric frequency up-converting energy harvester with rotating proof 
mass for human body applications, Sensors Actuators, A Phys. 206 (2014) 178-185. doi:10.1016/j.sna.2013.10.003.

[9] T. Xue, X. Ma, C. Rahn, S. Roundy, Analysis of Upper Bound Power Output for a Wrist-Worn Rotational Energy Harvester from Real-World Measured Inputs, J. Phys. Conf. Ser. 557 (2014) 12090. doi:10.1088/1742$6596 / 557 / 1 / 012090$.

[10] M. Pozzi, Magnetic plucking of piezoelectric bimorphs for a wearable energy harvester, Smart Mater. Struct. 25 (2016) 45008. doi:10.1088/0964-1726/25/4/045008.

[11] M. Pozzi, M.S.H. Aung, M. Zhu, R.K. Jones, J.Y. Goulermas, The pizzicato knee-joint energy harvester: characterization with biomechanical data and the effect of backpack load, Smart Mater. Struct. 21 (2012) 75023. doi:10.1088/0964-1726/21/7/075023.

[12] R. Lockhart, P. Janphuang, D. Briand, N.F. de Rooij, A wearable system of micromachined piezoelectric cantilevers coupled to a rotational oscillating mass for on-body energy harvesting, 2014 IEEE 27th Int. Conf. Micro Electro Mech. Syst. (2014) 370-373. doi:10.1109/MEMSYS.2014.6765653.

[13] M. Hayakawa, Electronic Wristwatch with Generator, U.S. Patent No.5,001,685. 19 Mar. 1991.

[14] Kinetron, Micro Generator System “26.4,” http://www.kinetron.eu/wp-content/uploads/2015/01/MGS-26.4.pdf, 2015 (accessed 19.09.2016). .

[15] T. Xue, S. Roundy, Analysis of Magnetic Plucking Configurations for Frequency Up-Converting Harvesters, in: J. Phys. Conf. Ser., 2015: pp. 1-5. doi:10.1088/1742-6596/660/1/012098.

[16] G. Akoun, J. Yonnet, 3D Analytical Calculation of the Forces Exerted between Two Cuboidal Magnets, IEEE Trans. Magn. MAG-20 (1984) 1962-1964. doi:10.1109/TMAG.1984.1063554.

[17] R. Ravaud, G. Lemarquand, S. Babic, V. Lemarquand, C. Akyel, Cylindrical magnets and coils: Fields, forces, and inductances, IEEE Trans. Magn. 46 (2010) 3585-3590. doi:10.1109/TMAG.2010.2049026.

[18] H.S. Choi, I.H. Park, S.H. Lee, Force calculation of magnetized bodies in contact using Kelvin's formula and virtual air-gap, IEEE Trans. Appl. Supercond. 16 (2006) 1832-1835. doi:10.1109/TASC.2005.864335.

[19] J.S. Agashe, D.P. Arnold, A study of scaling and geometry effects on the forces between cuboidal and cylindrical magnets using analytical force solutions, J. Phys. D. Appl. Phys. 42 (2009) 099801-099801. doi:10.1088/0022$3727 / 42 / 9 / 099801$.

[20] L.H. De Medeiros, G. Reyne, G. Meunier, Comparison of global force calculations on permanent magnets, IEEE Trans. Magn. 34 (1998) 3556-3559. doi:10.1109/20.717839.

[21] P. Pillatsch, E.M. Yeatman, A.S. Holmes, Magnetic plucking of piezoelectric beams for frequency up-converting 
energy harvesters, Smart Mater. Struct. 23 (2014) 25009. doi:10.1088/0964-1726/23/2/025009.

[22] H. Allag, J. Yonnet, Coulombian Model for 3D Analytical Calculation of the Torque Exerted on Cuboidal Permanent Magnets with Arbitrarly Oriented Polarizations, LDIA 2011 (8th Int. Conf. Linear Drives Ind. Appl. 2011 (2011) 1-5. http://hal.archives-ouvertes.fr/hal-00608059/.

[23] P. Elleaume, O. Chubar, J. Chavanne, Computing 3D magnetic fields from insertion devices, Proc. 1997 Part. Accel. Conf. (Cat. No.97CH36167). 3 (1997) 3509-3511. doi:10.1109/PAC.1997.753258.

[24] K.W. Yung, P.B. Landecker, D.D. Villani, An Analytic Solution for the Force Between Two Magnetic Dipoles, Magn. Electr. Sep. 9 (1998) 39-52. doi:10.1155/1998/79537.

[25] A. Erturk, D.J. Inman, Issues in mathematical modeling of piezoelectric energy harvesters, Smart Mater. Struct. 17 (2008) 65016. doi:10.1088/0964-1726/17/6/065016.

[26] M.A. Karami, J.R. Farmer, D.J. Inman, Parametrically excited nonlinear piezoelectric compact wind turbine, Renew. Energy. 50 (2013) 977-987. doi:10.1016/j.renene.2012.07.037.

[27] A. Erturk, D.J. Inman, Piezoelectric Energy Harvesting, John Wiley \& Sons, Ltd, Chichester, UK, 2011. doi:10.1002/9781119991151. 